\title{
A Study of Touch Typing Performance with Keyclick Feedback
}

\author{
Jin Ryong Kim and Hong Z. Tan \\ Haptic Interface Research Laboratory, Purdue University, West Lafayette, IN, USA
}

\begin{abstract}
The present study investigates the effect of haptic, auditory and visual keyclick feedback on touch typing performance using a zero-travel keyboard. We examine how local and global haptic keyclick feedback affect typing performance, and compare them with auditory keyclick feedback and visual feedback conditions. Our aim is to understand how sensory feedback during touch typing might improve user performance. Participants are asked to type required text shown on a computer screen and typing speed and error rates are recorded. The results show that the local haptic keyclick feedback condition leads to the highest typing speed with the lowest total error rate among all feedback conditions. We also find that the intensity of haptic feedback affects typing performance while the intensity of auditory feedback does not. Our findings provide useful design guidelines for improving touch typing performance on a zero-travel keyboard with sensory keyclick feedback information.
\end{abstract}

Keywords: flat keyboard, zero-travel keyboard, haptic feedback, auditory feedback, visual feedback, keyclick feedback, touch typing performance.

Index Terms: H.5.2 [INFORMATION INTERFACES AND PRESENTATION]: User Interfaces (D.2.2, H.1.2, I.3.6)Haptic I/O

\section{INTRODUCTION}

Recently, touchscreen devices such as smartphones and tablet PCs are getting extremely popular and permeating into our daily lives. People use touchscreen devices to interact with a variety of applications, play games, read and write emails and browse web pages. As people spend more time on their touchscreen devices, text entry for touchscreen devices becomes their daily activities. They use either an on-screen virtual keyboard or an external slim keyboard that can be a cover for the touchscreen device. The onscreen virtual keyboard is a software-based keyboard layout that is displayed on the touchscreen. In order to enter the text, the user presses on-screen keys with a finger or a stylus. It is easy to use and flexible in location and size. It is also easy to reconfigure since the layout of the virtual keyboard is based on software [1]. An alternate way that people use for text entry on touchscreen is to use a slim keyboard that can be easily connected to the device such as the Touch Cover for Microsoft Surface [2]. The Touch Cover keyboard is a thin-and-light flat keyboard that supports the full keyboard functionality for Surface. It also serves the dual role of a protection cover for the Surface touchscreen. The Surface Touch Cover uses pressure-sensing input technology for detecting key depresses and releases without any physical key travel. It also allows all the fingers to rest on the home row or anywhere else on

\{jessekim, hongtan\}@purdue.edu

IEEE Haptics Symposium 2014 23-26 February, Houston, Tx, USA 978-1-4799-3131-6/14/\$31.00 @2014 IEEE the keyboard without false triggering, thereby enabling ten-finger touch typing as people would do on any keyboard with mechanically-movable keys.

Although both on-screen keyboard and external thin keyboard are convenient to use, it is difficult to achieve a high level of typing performance due to at least two problems. The first problem is the lack of tactile feeling of the keyboard layout as compared to physical keyboards. This makes it more difficult for typists to locate their fingers on the home row and to feel the center and borders of each key, especially for on-screen keyboards. The inability to feel the locations of keys causes frequent gaze shifts between the keyboard and the text display area on the touchscreen and is a major source of degraded typing performance. One potential solution to this problem is to adaptively place a software keyboard under the typists' fingers. Findlater et al. [3] examined the unconstrained typing patterns of expert typists on a flat touch surface. They found that the key press locations remain relatively consistent within an individual and suggested personalization of keyboard layout as a way to support eyes-free touch typing in the absence of tactile cues for key locations.

The second problem associated with typing on a flat keyboard is the lack of confirmation for when a key has been pressed. Without the physical movement of keys on an on-screen keyboard or an external flat keyboard, there is no haptic confirmation cues for a typist to know for sure when a key press has been registered. Given that typing is a motor activity, the lack of "confirmed" keypress through haptic feedback can result in a significant performance degradation [4].

A number of solutions have been proposed to address this problem by providing tactile [5] [6] [7] [8] [9] [10], auditory [11] [12] [13], and visual [11] [14] [15] feedback. The first tactile interface on touchscreen was perhaps the Active Click [8]. It was designed to improve the input speed of touch panel operation in a noisy environment. Poupyrev et al. [9] [10] embedded a PDA with a TouchEngine [16] - a custom-designed haptic actuator using a thin piezoceramic film. They demonstrated that haptic feedback can be used effectively when interacting with small touch-based devices. In addition, there are several commercial attempts to create haptic keyclick feedback. Strategic Polymers Inc. [17] introduced AWAKE - an ultra-thin, light, and flexible zero-travel keyboard with haptic feedback. They used Electro-Mechanical Polymer (EMP) actuators that provide high electrostrictive response to the fingers when an electric field is applied. ViviTouch HD Feel [18] is a thin film component that is integrated into the touchscreen devices to simulate tactile sensations. It is based on an electroactive polymer (EAP) that consists of a thin layer of dielectric polymer film between two conductive electrodes. When it is activated, the film contracts in thickness and expands in area. Tactus Technology [19] invented a tactile user interface for touchscreen devices by erecting completely transparent, physical buttons on a flexible screen. It allows users to type or rest their fingers on the raised buttons and input data by pressing down on the buttons. They pumped fluid into dynamic microfluidic channels that allow the physical buttons to rise out of the touchscreen surface.

A haptic keyclick feedback signal can be local if the keyclick signal is felt by the typing finger only, or global if the keyclick 
signal is felt by all the fingers resting on the keyboard. Most keyclick feedback available in today's smartphones and tablets belong to global keyclick feedback. Such feedback is typically delivered by one tactor that moves the entire device (although see Poupyrev et al. [9] [10]). Global keyclick feedback may not be condusive to effective touch typing because all the fingers contacting the surface may receive the feedback at the same time. Local keyclick feedback may feel most natural as it simulates more closely how typing on a physical keyboard feels like.

The present study investigates how typing performance depends on the various types of sensory feedback that are available during typing. We examine whether haptic keyclick feedback signals lead to any improvement in typing performance, whether there is a difference between global and local haptic keyclick feedback, and how the effect of haptic feedback compare with that of auditory keyclick feedback that is more widely available in today's consumer products. We hasten to point out that we focus on haptic feedback signals that feel like a keyclick, as supposed to a vibration. A vibration is symbolic and its interpretation is context dependent. For example, a vibrating alert of a new text message felt during touch typing may lead the typist to believe that a false key entry has been registered. We built an apparatus with haptic feedback that feels like a keyclick: it feels distinctly different from a vibration. In addition, our keyboard apparatus is capable of delivering either global or local haptic keyclick feedback, thereby enabling the investigating of the effect of local vs. global haptic feedback on typing performance with the same testing apparatus. Finally, since visual feedback is typically available during typing on a touchscreen or flat keyboard, we also incorporate visual feedback in our experimental setup.

\section{Methods}

\subsection{Participants}

Twelve people (P1-P12; 6 males and 6 females; average age 28.8 years old, std. dev. 4.3 years old) participated in the experiments. Eleven of the participants were right-handed and one was ambidextrous by self-report. They were all touch typists who can type 50 or more words per minute (WPM). The participants were paid for their time.

\subsection{Apparatus}

\subsubsection{Keyboard Structure}

We constructed a zero-travel keyboard (a keyboard with keys that do not move when pressed) apparatus that can deliver local and global haptic keyclick feedback with controlled signal strengths by placing piezoelectric actuators under each key (see Fig. 1). We considered the following requirements for its design: i) touch input sensing; ii) haptic keyclick feedback output; and iii) isolation of haptic keyclick feedback among the keys. The top of the keyboard is an embossed rubber keyboard cover that is typically used to protect a qwerty keyboard from dust and spills. The keys can be felt tactily during touch typing.

For input sensing of key presses, the keyboard should be able to handle fast keystrokes entered by proficient touch typists. For this reason, we used the USB-based keyboard matrix circuit in a qwerty keyboard that is compatible with Apple's A1242 model for input sensing. The keys on this qwerty keyboard has the same size as those on a typical desktop keyboard and the same key layout. It does not have a numeric keypad. We removed the mechanical keys from the keyboard and the dome structure underneath them for key depression and release. We kept the keyboard matrix circuit inside the keyboard intact. The circuit consists of three transparent layers. The top and bottom layers have circular conductive buttons and they are insulated by the



Figure 1: (top panel) The zero-travel keyboard apparatus used in the present study, shown with the power supply and the highvoltage driver circuit (but not the High Voltage Amplifier, shown in Fig. 2). (bottom panels) Views of one key with the piezoelectric actuator (left), without the piezo (middle), and under the rubber keyboard cover (right), respectively.

middle layer. When a key is depressed in the original keyboard, the dome structure for each key is also depressed, causing the two conductive buttons to connect through the hole in the middle layer, thereby triggering a key entry. When the typing finger leaves the key, the spring force in the dome structure pushes the key upward to its original, disconnected position. After we removed the dome structure underneath the keys, we replaced them with $15 \times 15 \mathrm{~mm}$ foam pads for each key. The stiff foam pads prevent the keys from traveling, thereby effectively simulating a zero-travel keyboard. It also prevents false triggering of key presses from fingers resting on the home row. Furthermore, the foam pads can absorb any excessive vibration from the actuators, thereby providing maximum mechanical isolation to satisfy our third design requirement (see above). Two foam strips were placed underneath each side of the foam pad to prevent the key from wobbling when pressed (see the middle of the bottom panels in Fig. 1). The key press sensing mechanism remains the same as the original keyboard.

Figure 2 shows the entire keyboard system. For keyclick feedback output, we placed a piezoelectric (piezo for short) actuator (a 14-mm ceramic disk mounted concentrically on a 20 $\mathrm{mm}$ metal disk; by Murata, Inc, Japan; cut to fit within a $15 \times 15$ $\mathrm{mm}$ foam pad) between a key and its corresponding foam pad to generate haptic feedback. When a key press is sensed, a waveform is sent to the High Voltage Amplifier (top of Fig. 2) with a gain of 100 (Dual Channel High Voltage Precision Power Amplifier, Model 2350, TEGAM Inc., USA). The amplified signal is sent to the corresponding piezo actuator through one (or both) of two High Voltage Analog Switches, namely High Voltage Analog Switch 1 and High Voltage Analog Switch 2 (HV20822, Supertex Inc., USA; see the middle of Fig. 2), to deliver keyclick feedback. We used the two audio channels (stereo: left and right) from the PC to generate haptic waveform signals. The two audio channels have the same signal strengths and their intensity is controlled by the sound volume. These two waveform signals are sent to Channel 1 Input and Channel 2 Input of the High Voltage Amplifier, respectively. The amplified waveform signals are then sent through one (or both) of the High Voltage Analog Switches. 


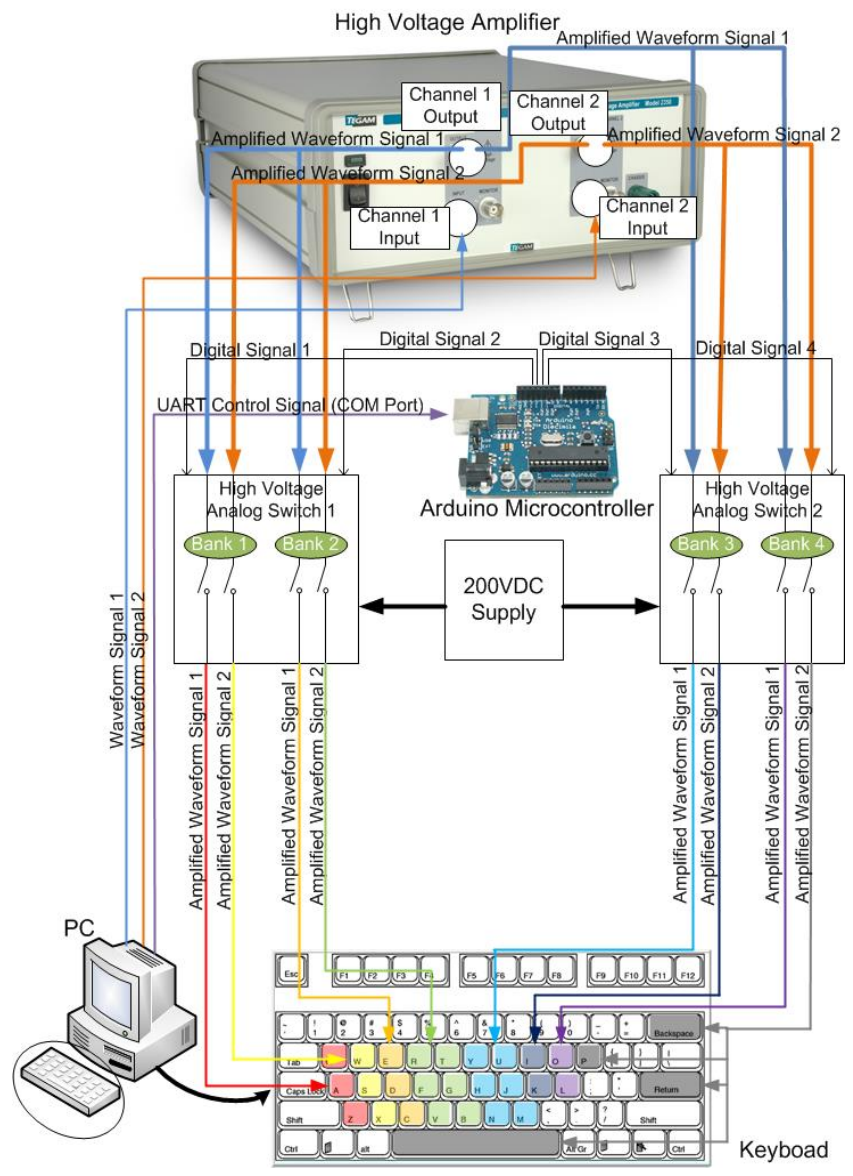

Figure 2: Illustration of the keyboard system. See text for more details.

Both High Voltage Analog Switches are controlled by an Arduino Microcontroller unit (ATmega168, Clock Speed: $16 \mathrm{MHz}$, Arduino Diecimila, Italy), which is in turn controlled by a keyboard agent to independently drive the piezo actuators to route the amplified waveform signals to each key. The keyboard agent is a middleware software that monitors the key input and generates the waveform signal while controlling the microcontroller unit. The keys are arranged into eight groups, with key(s) in the same group being assigned to the same finger during touch typing. All keys in the same group received the same amplified waveform signal. For example, ' $q$ ', ' $a$ ', and ' $z$ ' keys are in the same group and they receive the same Amplified Waveform Signal 1 (colored red in Fig. 2) from Channel 1 Output of the High Voltage Amplifier, routed through Bank 1 in the High Voltage Analog Switch 1. In the same manner, 'i' and ' $k$ ' keys are in the same group and they receive the Amplified Waveform Signal 2 (colored dark blue in Fig. 2) from Channel 2 Output of the High Voltage Amplifier through Bank 3 in the High Voltage Analog Switch 2. When 'a' is pressed in the local haptic keyclick feedback condition, Waveform Signal 1 from the PC is sent to Channel 1 Input. Amplified Waveform Signal 1 is then sent to High Voltage Analog Switch 1. At the same time, the Arduino Microcontroller unit turns on the corresponding switch in Bank 1 of the High Voltage Analog Switch 1 to route the Amplified Waveform Signal 1 (colored red in Fig. 2) to the group of keys containing ' $a$ ' to deliver a local haptic keyclick feedback. For global haptic feedback, we send both Waveform Signals 1 and 2 to the High Voltage Amplifier and turn on all the switches in High Voltage Analog Switches 1 and 2. By having two channels in the High Voltage Amplifier, we can control the actuators individually with a minimum number of switches. The waveform that is sent to each piezo consists of one cycle of a raised sinusoidal pulse at 500 $\mathrm{Hz}$ to simulate a "crisp" keyclick (cf. [20]).

\subsubsection{Measurement of Keyclick Feedback Delay}

An important specification of our keyboard system is the delay between a key press and the resultant haptic keyclick feedback. This delay was measured with a triaxial accelerometer (Kistler KShear Accelerometer Type 8794A, Kistler, USA). We attached the accelerometer on top of the "a" key of the keyboard apparatus and pressed on the accelerometer to measure the time difference between the moments when we pressed the key and when the haptic feedback was activated. Out of 10 trials of key presses, the delay ranged from 48.6 to $68.4 \mathrm{~ms}$, the mean was $58.7 \mathrm{~ms}$ and the standard deviation was $6.6 \mathrm{~ms}$.

To determine whether the measured delay was acceptable, we conducted a perception experiment to estimate the human detection threshold of such a delay with two participants. The method of adjustment was used [21]. The participants were asked to adjust the delay of the keyclick feedback signal until they could barely feel any delay. The step size of each adjustment was $5 \mathrm{~ms}$ and it was increased from $0 \mathrm{~ms}$ in software. The total delay was the sum of software-induced delay and the minimum delay as reported above. The measured software-induced delay averaged $40.8 \mathrm{~ms}$. Therefore, the estimated human detection threshold for total delay was approximately $99.5(40.8+58.7) \mathrm{ms}$.

Our results can be compared to those reported by Kaaresoja et al. [22]. Their participants typed a short sentence shown on a display using a virtual keyboard with haptic feedback. They varied the feedback delay from $18 \mathrm{~ms}$ to $118 \mathrm{~ms}$ in 20 -ms steps and measured the average typing time and error rate. They reported that a single factor ANOVA analysis did not show any statistical difference among the feedback delays. They also reported that half of the participants (out of 12) noticed a delay between $98 \mathrm{~ms}$ and $118 \mathrm{~ms}$, and the rest of the participants did not notice any delay at all. Their result was consistent with our measured detection threshold of $99.5 \mathrm{~ms}$. Therefore, the hardware delay from our keyboard apparatus (48.6 to $68.4 \mathrm{~ms}$ ) was deemed acceptable (i.e., below human detection threshold) for the present study.

We further quantified the signal attenuation between the haptic feedback measured on the key being pressed and that on a neighboring key. We placed an accelerometer on the ' $a$ ' key and measured the acceleration profile while activating the keyclick feedback on the 'a' key for 10 times. We then placed the accelerometer on the 's' key and measured the acceleration profile while activating the keyclick feedback on the 'a' key for 10 times. The average peak acceleration was $1.75 \mathrm{~g}$ and $0.17 \mathrm{~g}$ for the ' $\mathrm{a}$ ' and 's' keys, respectively. The corresponding attenuation threshold was therefore $20.25 \mathrm{~dB}\left(20 \times L O G_{10}(1.75 / 0.17)\right)$. This result can be compared to the attenuation threshold reported by Kim et al. [23] in a masking study. They measured the attenuation threshold between two fingers (a typing finger and a non-typing finger) with different finger combinations on one or two hands. Their results indicated that an attenuation threshold of $19.5 \mathrm{~dB}$ (for fingers on different hands) or $11.5 \mathrm{~dB}$ (for fingers on the same hand) is required for the haptic keyclick feedback signal on the non-typing finger to be masked by that on the typing finger. Therefore, our measured attenutation of $20.25 \mathrm{~dB}$ was sufficiently large to ensure that when only one piezo is activated, a local haptic keyclick feedback signal is felt on the typing finger only, and not on any other fingers resting on our keyboard apparatus. 


\subsection{Experimental Conditions}

We conducted a multi-finger touch typing experiment to measure typing performance on a flat keyboard with eight feedback conditions (see Table 1). Two sets of visual feedback conditions, asterisk and letter sets, were used. In the asterisk set, each key press was displayed visually as an asterisk character. This minimal visual feedback condition provides similar amount of feedback information as a haptic or auditory keyclick feedback signal (i.e., a key has been pressed, but not the key's identity). The asterisk visual feedback condition is therefore useful for a fair comparison of the effects of visual, auditory and haptic keyclick feedback. The letter set not only acknowledges a key click but also shows the letter that was just typed. This feedback condition is commonly used in real-world typing scenarios and will therefore be evaluated as well. In all the conditions, visual feedback was always available, either as an asterisk or as a letter, since typists always get visual feedback while they type and it is difficult to type without visual feedback.

As shown in Table 1, the same four haptic/auditory feedback conditions were used for either asterisk or letter visual feedback conditions, yielding a total of eight experimental conditions. The first haptic/auditory condition was local haptic keyclick feedback (localH for short) where only the key being pressed is activated to generate the keyclick feedback for each keystroke, and no auditory feedback was available. The second condition was global haptic keyclick feedback (globalH for short) where all keys were activated for each keystroke, and no auditory feedback was available. A pilot test using the method of adjustment [21] was conducted to equalize the perceived keyclick feedback intensity for local and global haptic feedback conditions. Results from three participants indicated that a signal amplitude of $60 \%$ sound volume for global haptic keyclick feedback felt to be as strong as a signal amplitude of $100 \%$ sound volume for local haptic keyclick feedback. The third condition was auditory keyckick feedback where a beep sound was played for each keystroke, and no haptic feedback was available. In the fourth condition, no haptic nor auditory keyclick feedback was provided.

Table 1. Experimental Conditions

\begin{tabular}{ccc}
\hline \hline Visual & Haptic & Auditory \\
\hline \hline \multirow{3}{*}{ asterisk } & localH & none \\
\cline { 2 - 3 } & globalH & none \\
\cline { 2 - 3 } & none & beep \\
\cline { 2 - 3 } & none & none \\
\hline \multirow{3}{*}{ letter } & localH & none \\
\cline { 2 - 3 } & globalH & none \\
\cline { 2 - 3 } & none & beep \\
\cline { 2 - 3 } & none & none \\
\hline \hline
\end{tabular}

\subsection{Procedures}

Before we conducted the main experiment, we measured each participant's baseline typing performance using a regular desktop keyboard (Dell USB Keyboard Model L100, Dell Inc, USA) with both asterisk and letter visual feedback. During the main experiment, we asked the participants to keep their fingers on the home row at all times and monitored their compliance with a webcam to make sure that their fingers rested on the home row as touch typists would. We covered the keyboard apparatus with a black cloth during the main experiment to block the view of the fingers and the keyboard apparatus. All participants listened to pink noise from an earphone and in addition wore a circumaural noise-reduction headphone (Peltor H10A Optime105 with $29 \mathrm{~dB}$ attenuation, 3M Corporation, USA) to block any auditory cues from the experimental apparatus. For the conditions involving auditory feedback, a beep sound was played through the earphone instead of pink noise. We evaluated typing performance using the typing test program called TextTest and StreamAnalyzer [24]. The participants typed twenty five phrases randomly selected from the MacKenzie phrase set [25]. Out of the twenty five phrases, the first five of the phrases were provided as practice. We asked the participants to type as fast and accurately as possible. We allowed a break period between experimental conditions in order to avoid any typing fatigue. The experiment took between 1.5 hours to 1 hour and 45 minutes.

During the experiment, we used the policy of "recommending" error corrections such that the participants were asked to correct any errors they detected or felt that they made. It should be noted that this did not guarantee that the participants corrected all the errors, especially during the asterisk visual feedback condition where the participants did not know which key(s) had been pressed. Total error rate, which were separated into the two components of corrected and uncorrected error rates, are usually used with the recommended error correction policy [26].

Prior to the main experiment, we ran a pilot test to investigate how intensity of the haptic and auditory keyclick feedback affect the typing performance. Three of the twelve participants took part in the pilot study by conducting two additional conditions in the asterisk set: i) global keyclick feedback with high intensity; and ii) auditory keyclick feedback with low intensity. The results were compared with those of the global haptic feedback and the beep auditory feedback conditions from the main experiment in the asterisk visual feedback set. All three participants typed faster with the higher intensity global haptic keyclick feedback (60.8 WPM) than with the lower intensity used in the main experiment (52.0 WPM). However, they didn't type any faster with the higher intensity auditory beep feedback used in the main experiment (53.6 WPM) than with the lower intensity used in the pilot study (55.6 WPM). We hypothesized that a weaker haptic keyclick feedback may have caused the participants to press down harder on the keyboard apparatus and therefore slowed them down with typing speed. The effect of haptic feedback intensity on typing speed deserves further investigation in the future.

\subsection{Data Analysis}

We measured several performance metrics for the experiment. Typing speed is measured in word per minute (WPM) and it is calculated as

$$
\mathrm{WPM}=\frac{|T|-1}{S} \times 60 \times \frac{1}{5}
$$

where $|\mathrm{T}|$ is the length of the transcribed string in number of characters and $\mathrm{S}$ is the time in seconds from the first keystroke to the last [27]. We subtract ' -1 ' from $|\mathrm{T}|$ to remove the time for typing the first character from the calculation of inter-character speed. The average length of a "word" is reported to be 5 characters [28] and we multiply the speed by 60 to convert the unit from words per second to words per minute.

The second metric that we measured is keystroke per character (KSPC) [29]. KSPC is widely used and it is the ratio of the length of input string to the length of transcribed text string.

$$
\mathrm{KSPC}=\frac{|I S|}{|T|}
$$

where $|\mathrm{IS}|$ is the length of the input string. KSPC considers the cost of committing errors and fixing them so it provides a general idea of how efficient the typing process is [26]. KSPC is 1 for the ideal case of no error corrections. It is greater than 1 when errors are found and corrected. 
The next metric that we measured is called the total error rate [30]:

$$
\text { Total Error Rate }=\frac{I N F+I F}{C+I N F+I F} \times 100 \%
$$

where INF (Incorrect Not Fixed) is the number of wrongly-typed characters that are not fixed, IF (Incorrect Fixed) is the number of wrong characters fixed, and $C$ (Correct) is the number of correctly-typed characters. The total error rate shows the ratio of the total number of incorrect characters (whether they are corrected or not) over the total number of correct and incorrect characters combined. The total error rate is further divided into corrected and uncorrected error rates as follows:

$$
\begin{gathered}
\text { Corrected Error Rate }=\frac{I F}{C+I N F+I F} \times 100 \% \\
\text { Uncorrected Error Rate }=\frac{I N F}{C+I N F+I F} \times 100 \%
\end{gathered}
$$

The performance metrics were analyzed with an analysis of variance (ANOVA) and post hoc Tukey tests, all at a significance level of $\alpha=.05$.

\section{Results}

Figure 3 shows the experimatal results for eleven of the twelve participants. We had to remove one participant's data from analyses because the participant did not attempt to correct any errors despite repeated reminders from the experimenter. Figure 3(a) shows the average WPM for the participants. In the asterisk visual feedback condition set (asterisk set for short), the average WPM is $65.6,55.6,52.4,51.1$, and 43.1 words per minute for the physical keyboard, localH, globalH, beep, and none conditions, respectively. In the letter visual feedback condition set (letter set for short), the average WPM is $67.0,55.1,51.8,50.2$, and 46.3 words per minute for the same set of conditions, respectively. In general, typing speed is the highest in the physical keyboard condition ( $\mu=66.3$ words per minute) and lowest in the none condition ( $\mu=44.7$ words per minute). There is a trend that WPM for the localH keyclick feedback conditions are higher than those for the globalH keyclick feedback conditions, which in turn were higher than those for the beep feedback conditions. A one-way ANOVA confirms that feedback condition was indeed a significant factor for WPM $\left(\mathrm{F}_{4,50}=8.97, p<.0001\right.$ for the asterisk set; $\mathrm{F}_{4,50}=6.09, p<.0005$ for the letter set). A post hoc Tukey test for the astersk set shows three groups: the physical keyboard and localH conditions ( $\mu=60.6$ words per minute); the localH, globalH, and beep conditions ( $\mu=53.1$ words per minute); and the globalH, beep, and none conditions ( $\mu=48.9$ words per minute). A post hoc Tukey test for the letter set shows two groups: the physical keyboard and localH conditions $(\mu=61.1$ words per minute); and the localH, globalH, beep, and none conditions ( $\mu=50.8$ words per minute). Finally, it is interesting to see that typing speed is consistent under the asterisk and letter visual feedback conditions, indicating that the information content of the visual feedback did not affect typing speed significantly. This is further confirmed with a one-way ANOVA that visual feedback condition was not a statistically significant factor for WPM $\left(\mathrm{F}_{1,108}=.05, p<.8289\right)$.

Figure 3(b) shows the average KSPC. On average, KSPC for the asterisk set is $1.08,1.10,1.09,1.12$, and 1.19 for the physical keyboard, localH, globalH, beep, and none conditions, respectively. KSPC for the letter set is $1.12,1.17,1.19,1.19$, and 1.27 for the same set of conditions, respectively. The physical keyboard condition shows the lowest KSPC whereas the none condition shows the highest KSPC, for both visual condition sets.



(a) Average WPM

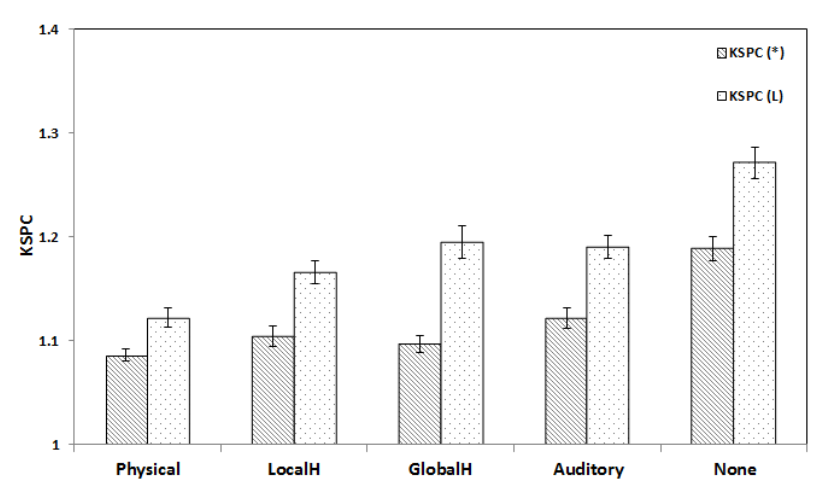

(b) Average KSPC

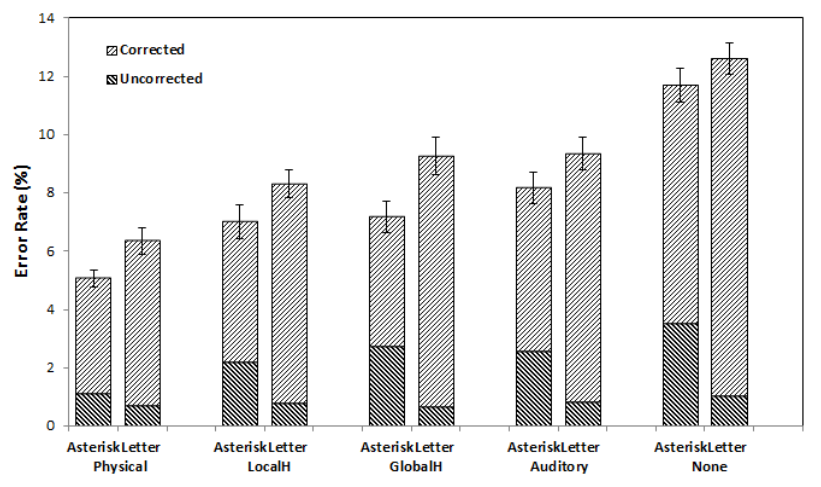

(c) Average corrected and uncorrected error rates. The total height of each stacked bar shows the total error rate as the sum of corrected and uncorrected error rates

Figure 3: Experimental results. Error bars indicate standard error.

In general, KSPCs in the letter set are higher than those in the asterisk set across the conditions. This is likely due to the fact that the participants were able to detect more errors in the letter visual feedback condition and tried to correct them, thereby generating more input characters. A one-way ANOVA confirms that the visual feedback condition was a significant factor for KSPC $\left(\mathrm{F}_{1,108}=17.82, p<.0001\right)$. Another one-way ANOVA confirms that feedback conditions was a significant factor for $\mathrm{KSPC}\left(\mathrm{F}_{4,50}=4.37\right.$, $p<.0041$ for the asterisk set; $\mathrm{F}_{4,50}=4.18, p<.0054$ for the letter set). In the asterisk set, a post hoc Tukey test shows two groups: the physical keyboard, localH, globalH, and beep feedback conditions $(\mu=1.10)$; and beep and none conditions $(\mu=1.16)$. A post hoc Tukey test for the letter set shows two groups: all but the none 
condition $(\mu=1.17)$, and all but the physical keyboard condition $(\mu=1.21)$.

The data in Figures 3(a) and 3(b) show, together, that typing performance generally deteriorated (in terms of decreased WPM and increased KSPC) from physical keyboard to flat keyboard, and from localH, globalH, beep to none condition. The results of the statistical analyses, however, are more nuanced.

Figure 3(c) shows the error rate results of uncorrected (lower bars) and corrected (upper bars) error rates, with the total height representing the total error rate. In the asterisk set, the uncorrected error rate is $1.1,2.2,2.7,2.6$, and $3.5 \%$ for the physical keyboard, localH, globalH, beep, and none conditions, respectively. In the letter set, the uncorrected error rate is $.7, .8, .6, .8$, and $1.0 \%$ for the physical keyboard, localH, globalH, beep, and none conditions, respectively. A visual inspection shows clearly that the uncorrected error rates are much higher in the asterisk set $(\mu=2.4 \%)$ than in the letter set $(\mu=.8 \%)$. In the asterisk set, there is a general trend of decreased performance (i.e., increased uncorrected error rate) from the physical keyboard to the flat keyboard and from the haptic/auditory feedback conditions to the none condition, although the differences were not statistically significant. A post hoc Tukey test for the asterisk set shows two groups: all but the none condition $(\mu=2.7 \%)$, and all but the physical keyboard condition $(\mu=2.1 \%)$. In contrast, in the letter set, the uncorrected error rates appear to remain constant across the conditions. A one-way ANOVA confirmed that feedback condition was not a significant factor for uncorrected error rate $\left(\mathrm{F}_{4,50}=2.24, p=.078\right.$ for the asterisk set; $\mathrm{F}_{4,50}=.39, p=.8165$ for the letter set). A post hoc Tukey test for the letter set shows only one group, confirming that the uncorrected error rate is not significantly different across the conditions tested. Overall, the uncorrected error rate results suggest that the uncorrected error rate depends greatly on the visual feedback. A one-way ANOVA shows that visual feedback condition was indeed a significant factor for uncorrected error rate $\left(\mathrm{F}_{1,108}=31.01, p<.0001\right)$. The letter visual feedback presumably allowed the participants to be more aware of errors and consequently resulted in significantly lower uncorrected error rates.

The upper bars in Figure 3(c) show the average corrected error rate. In the asterisk set, the average corrected error rate is 4.0, 4.9, 4.5, 5.6, and $8.2 \%$ for the physical keyboard, localH, globalH, beep and none conditions, respectively. In the letter set, the average corrected error rate is 5.7, 7.6, 8.7, 8.6, and $11.6 \%$ for the same set of conditions, respectively. A visual inspection shows that the letter visual feedback condition resulted in more corrected errors than the asterisk condition. This is confirmed by a one-way ANOVA showing that the visual feedback condition was indeed a significant factor for corrected error rate $\left(\mathrm{F}_{1,108}=19.58, p<.0001\right)$. There is a general trend of lower corrected error rate in the physical keyboard condition and higher corrected error rate in the none condition for both visual condition sets. A one-way ANOVA confirms that feedback condition was a significant factor for corrected error rate $\left(\mathrm{F}_{4,50}=3.87, p=.0081\right.$ for the asterisk set; $\mathrm{F}_{4,50}=4.07, p<.0062$ for the letter set). A post hoc Tukey test for the asterisk set shows two groups: all but the none condition $(\mu=4.7 \%)$ and the localH, beep and none conditions $(\mu=6.2 \%)$. A post hoc Tukey test for the letter set shows two groups: all but the none condition $(\mu=7.6 \%)$ and all but the physical keyboard condition $(\mu=9.1 \%)$.

The combined lower and upper bars in Figure 3(c) show the average total error rates, which are the combined results from the uncorrected and corrected error rates. In the asterisk set, the average total error rate is $5.1,7.0,7.2,8.2$, and $11.7 \%$ for the physical keyboard, localH, globalH, beep and none conditions, respectively. In the letter set, the average total error rate is $6.3,8.3$, $9.3,9.4$, and $12.6 \%$ for the same set of conditions, respectively. It appears that the letter visual feedback condition resulted in slightly higher error rates than the asterisk condition, although the difference is not statistically significant $\left(\mathrm{F}_{1,108}=2.93, p=.0898\right)$. It is clear that the participants made the least errors with physical keyboards and the most errors under the none condition. This is confirmed by a one-way ANOVA on total error rate showing that feedback condition was a significant factor $\left(\mathrm{F}_{4,50}=5.12, p=.0015\right.$ for the asterisk set; $\mathrm{F}_{4,50}=3.93, p=.0075$ for the letter set). A post hoc Tukey test for the asterisk set shows two groups: all but the none condition $(\mu=6.9 \%)$ and beep and none conditions $(\mu=9.9 \%)$. This means that the total error rate for the none condition was significantly higher than those for the other conditions (except for the beep ondition). A post hoc Tukey test for the letter set shows two less distinct groups: all but the none condition $(\mu=8.3 \%)$ and all but the physical keyboard condition $(\mu=9.9 \%)$.

Overall, feedback condition was a significant factor for WPM, $\mathrm{KSPC}$, uncorrected error rate, corrected error rate and total error rate, except for the uncorrected error rate in the letter set. The visual feedback condition was a significant factor for KSPC, uncorrected error rate, and corrected error rate, but not for WPM or total error rate.

\section{Discussion AND Conclusion}

The present study investigated the effects of haptic, auditory and visual feedback on typing performance using a zero-travel keyboard. We hypothesized that people will perform better in terms of higher typing speed and lower error rate when keyclick feedback is provided. We further hypothesized that local haptic feedback might outperform global haptic feedback in typing speed and/or typing error since the local haptic feedback condition resembles more closely the availability of keyclick feedback on a physical keyboard.

Our results show that the participants performed the best with the physical keyboard. This was expected since the physical keyboard provides rich multisensory feedback and is most familiar to touch typists. With the physical keyboard, the participants typed with the highest speed (WPM), the lowest $\mathrm{KSPC}$, and the lowest error rate (uncorrected, corrected, and total error rates). It is also clear that haptic keyclick feedback and auditory beep sound feedback improved the typing performance, as compared to the none (no haptic nor auditory feedback) condition. Additional findings are noted below.

First, the typing speed for the local keyclick feedback condition was highest among all feedback conditions on a zero-travel keyboard apparatus. The total error rate for the local keyclick feedback condition was also the lowest among all feedback conditions. KSPC for the local feedback condition was the second lowest for the asterisk set and the lowest for the letter set. The uncorrected error rate was the lowest among all feedback conditions for the asterisk set and the second lowest for the letter set. The corrected error rate was the second lowest for the asterisk set and the lowest for the letter set. Therefore, although the local haptic feedback condition did not "win" in all conditions tested, it came very close to having the highest typing speed and lowest typing error rate. However, only one participant noticed the difference between global and local keyclick feedback. Given the increased complexity associated with the construction of a zerotravel keyboard with local keyclick feedback, the potential performance gain associated with local feedback need to be carefully weighed against materials and apparatus cost.

Second, we found through preliminary testing that haptic feedback intensity affects the typing performance whereas auditory feedback intensity does not. The effect of haptic feedback intensity on performance is mainly due to the fact that people press down harder with weaker haptic feedback, yielding a 
lower typing speed. Our finding is consistent with those of Kaaresoja et al. [22]. In their study, some of the participants made more errors with the virtual keypad with longer delay and they pressed harder on the buttons on a touchscreen. In our study, since only three participants and two intensity levels were tested, this issue needs to be further investigated with more participants and more intensity levels in the future.

Third, the type of visual feedback did not significantly affect typing speed or total error rate. However, the average KSPC and corrected error rate were significantly higher and uncorrected error rate was significantly lower in the letter set than in the asterisk set. It thus appears that knowing that a key has been pressed but not necessarily its identity was sufficiently useful to improve the typing speed and to reduce the overall typing errors, but knowing which key has just been pressed allowed the participants to correct more typing errors, thereby increasing $\mathrm{KSPC}$ and corrected error rate while reducing uncorrected error rate.

Although not every result obtained in the present study reached statistical significance, a majority of the participants demonstrated improved typing performance with sensory feedback. It shows that it was useful for the typists to know that a key press has been registered by a touchscreen device. Furthermore, the information on which key has been pressed played an important role in improving typing. Among the different types of feedback available on our zero-travel keyboard apparatus and assuming the availability of visual feedback at all times, local keyclick feedback showed the highest typing speed and the lowest KSPC, corrected error rate and total error rate. It also showed the second lowest uncorrected error rate among the different types of feedback conditions. However, the design choice should be carefully evaluated due to the complexity of local keyclick feedback. Our findings provide useful engineering specifications for designing haptic, auditory, and visual feedback of zero-travel keyboards. In the future, we need to investigate how intensity of the haptic feedback affects typing performance with more intensity levels and more participants. We will also investigate how keyclick feedback and key identity information can be beneficial, respectively, to improving touch typing performance and experience.

\section{ACKNOWLEDGMENT}

The authors thank Professor Jacob O. Wobbrock of University of Washington for sharing his source code of TextTest. This work was partially supported by a grant from Microsoft Research.

\section{REFERENCES}

[1] U. Hinrichs, M. Hancok, C. Collins, and S. Carpendale, "Examination of Text-Entry Methods for Tabletop Display," in Second Annual IEEE International Workshop on Horizontal Interactive Human-Computer System, pp. 105-112, 2007.

[2] Surface Touch Covers: http://www.microsoft.com/surface/enus/accessories/touch-cover

[3] L. Findlater, J. Wobbrock, and D. Wigdor, "Typing on Flat Glass: Examining Ten-Finger Expert Typing Patterns on Touch Surfaces," in ACM CHI 2011, pp. 2453-2462, 2011.

[4] M. Crump and G. Logan, "Warning: This Keyboard will Deconstruct - The Role of the Keyboard in Skilled Typewriting," Psychonomic Bulletin \& Review, Volume 17, pp. 394-399, 2010.

[5] S. Lee and S. Zhai, "The Performance of Touch Screen Soft Buttons," in ACM CHI 2009, pp. 309-318, 2009.

[6] E. Hoggan, S. Brewster, and J. Johnston, "Investigating the Effectiveness of Tactile Feedback for Mobile Touchscreens," in ACM CHI 2008, pp. 1573-1582, 2008.

[7] S. Brewster, F. Chohan, and L. Brown, "Tactile Feedback for Mobile Interaction," in ACM CHI 2007, pp. 159-162, 2007.
[8] M. Fukumoto and T. Sugimura, "Active Click: Tactile Feedback for Touch Panels," in ACM CHI 2001, pp. 121-122, 2007.

[9] I. Poupyrev, S. Maruyama, and J. Rekimoto, "Ambient Touch: Designing Tactile Interfaces for Handheld Devices," in UIST'02: ACM Symposium on User Interface Software and Technology, pp. 51-60, 2002

[10] I. Poupyrev and S. Maruyama, "Tactile Interfaces for Small Touch Screens," in UIST'03: ACM Symposium on User Interface Software and Technology, pp. 217-220, 2003.

[11] P. Majaranta, I. MacKenzie, A. Aula, and K. Räihä, "Auditory and Visual Feedback during Eye Typing," in $A C M$ CHI 2003, pp. 766$767,2003$.

[12] H. Tinwala and I. MacKenzie, "Eyes-free Text Entry on a Touchscreen Phone," in Science and Technology for Humanity, pp. $83-88,2009$

[13] G. Bender, "Touch Screen Performance as a Function of the Duration of Auditory Feedback and Target Size," Doctor of Philosophy Doctoral Dissertation, College of Liberal Arts and Sciences, Wichita State University, The Graduate School of Wichita State University, 1999.

[14] L. Magnien, J. Bouraoui, and N. Vigouroux, "Mobile Text Input with Soft Keyboards: Optimization by Means of Visual Cues," in MobileHCI 2004, pp. 337-341, 2004.

[15] T. Paek, K. Chang, I. Almog, E. Badger, and T. Sengupta, "Multimodal Feedback and Guidance Signals for Mobile Touchscreen Keyboards," in TechReport (MSR-TR-2010-76), 2010.

[16] I. Poupyrev, S. Maruyama, and J. Rekimoto, "TouchEngine: A Tactile Display for Handheld Devices," in ACM CHI 2002, pp. 644645, 2002.

[17] Strategic Polymers Inc: http://www.strategicpolymers.com

[18] Artificial Muscle Inc - A Bayer MaterialScience Company: http://www.vivitouch.com

[19] Tactus Technology Inc: http://www.tactustechnology.com

[20] H. Chen, J. Park, S. Dai, and H. Tan, "Design and Evaluation of Identifiable Key-click Signals for Mobile Devices," IEEE Transactions on Haptics, Volume 4, pp. 229-241, 2011.

[21] G. Gescheider, Psychophysics: Method, Theory, and Application. Hillsdale, New Jersey: Lawrence Erlbaum, 1985.

[22] T. Kaaresoja, E. Anttila, and E. Hoggan, "The Effect of Tactile Feedback Latency in Touchscreen Interaction," in WHC'11: IEEE World Haptics Conference, pp. 65-70, 2011.

[23] J. Kim, X. Dai, X. Cao, C. Picciotto, D. Tan, and H. Tan, "A Masking Study of Key-Click Feedback Signals on a Virtual Keyboard," in Eurohaptics 2012, pp. 247-257, 2012.

[24] J. Wobbrock and B. Myers, "Analyzing the Input Stream for Character-Level Errors in Unconstrained Text Entry Evaluations," ACM Transactions on Computer-Human Interaction, Volume. 13, pp. 458-489, 2006.

[25] I. MacKenzie and R. Soukoreff, "Phrase Sets for Evaluating Text Entry Techniques," in Extended Abstracts of ACM CHI 2003, pp. 754-755, 2003.

[26] A. Sabbir and W. Stuerzlinger, "Analysis of Text Entry Performance Metrics," in IEEE Toronto International Conference on Science and Technology for Humanity Toronto, ON, 2009.

[27] I. MacKenzie. A Note on Calculating Text Entry Speed. Available: http://www.yorku.ca/mack/RN-TextEntrySpeed.html

[28] H. Yamada, "A Historical Study of Typewriters and Typing Methods: From the Position of Planning Japanese Parallels," Journal of Information Processing, Volume 2, pp. 175-202, 1908.

[29] R. Soukoreff and I. MacKenzie, "Measruing Errors in Text Entry Tasks: An Application of the Levenshtein String Distance Statistic," in ACM CHI 2001, pp. 319-320, 2001.

[30] R. Soukoreff and I. MacKenzie, "Metrics for Text Entry Research: An Evaluation of MSD and KSPC, and a New Unified Error Metric," in ACM CHI 2003, pp. 113-120, 2003. 